\title{
Dynamic focusing with high-quality-factor metalenses
}

Elissa Klopfer,${ }^{1 *}$ Mark Lawrence, ${ }^{1}$ David R. Barton, III, ${ }^{1}$ Jefferson Dixon, ${ }^{2}$ Jennifer A. Dionne $e^{1,3^{*}}$

${ }^{1}$ Stanford University, Department of Materials Science and Engineering

${ }^{2}$ Stanford University, Department of Mechanical Engineering

${ }^{3}$ Stanford University, Department of Radiology

*Corresponding author: eklopfer@stanford.edu, jdionne@stanford.edu 


\section{Supporting Information Section 1: Computational Model}

We leveraged the finite element solver COMSOL Multiphysics to perform frequency domain calculations of our metalenses. First, 2D simulations were performed to model a slice of an infinite series of nanobars spaced $700 \mathrm{~nm}$ apart, $700 \mathrm{~nm}$ in height, with constant width varied from $100 \mathrm{~nm}$ to $400 \mathrm{~nm}$. Floquent boundaries were used on either side of a nanobar and port boundaries, polarized into the plane, were used above and below the nanobars. This simulation was used as a basis for determining the phase delay associated with different width nanobars, shown in Figure S1a. Figure S1b and c show the individual bar widths, and their phase, chosen to approximate the parabolic phase profile defined in the main text.
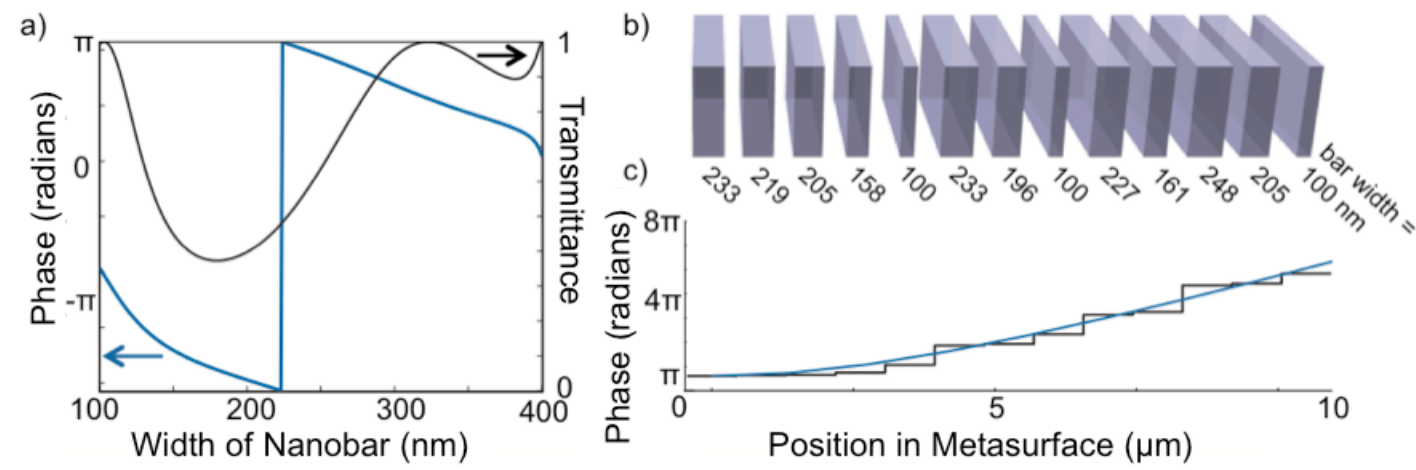

Figure S1: a) Transmittance (black) and phase shift (blue) associated with a normally incident, y-polarized plane wave on a series of bars of height $700 \mathrm{~nm}$, spaced $700 \mathrm{~nm}$ apart, with width varying from 100 to $400 \mathrm{~nm}$, b) Schematic of one half of the symmetric metalens with the various nanobars labeled with their associated widths, c) Parabolic phase profile calculated for a metalens with a focal length of $4 \mu \mathrm{m}$ operating at $\lambda=1500$ 
nm, as well as the approximation of this parabolic curve by a series of discrete phase steps and the associated antenna widths.

To model this metalens, both two-dimensional (2D) and three-dimensional (3D) simulations were performed. The 2D simulations were leveraged to calculate the guided modes in the unperturbed silicon nanobars of the metalens via mode analysis. The guided modes calculated are shown in Figure S2 for the second from the center nanobar (w=219 $\mathrm{nm}$ in purple), the third from the center nanobar ( $\mathrm{w}=205 \mathrm{~nm}$ in blue), the fourth from the center nanobar ( $\mathrm{w}=158 \mathrm{~nm}$ in green). Figure S2a also shows the theoretical band folding associated with a periodic perturbation of $600 \mathrm{~nm}$ in all cases. Figure S2b shows the band folding associated with one set of nanobars $(\mathrm{w}=219 \mathrm{~nm}$ in purple) being notched at 600 $\mathrm{nm}$, while another set of nanobars $(\mathrm{w}=158 \mathrm{~nm}$ in green) are notched with a period of 675 $\mathrm{nm}$, such that both achieve a guided mode resonance (GMR) at a wavelength of 1500 nm.
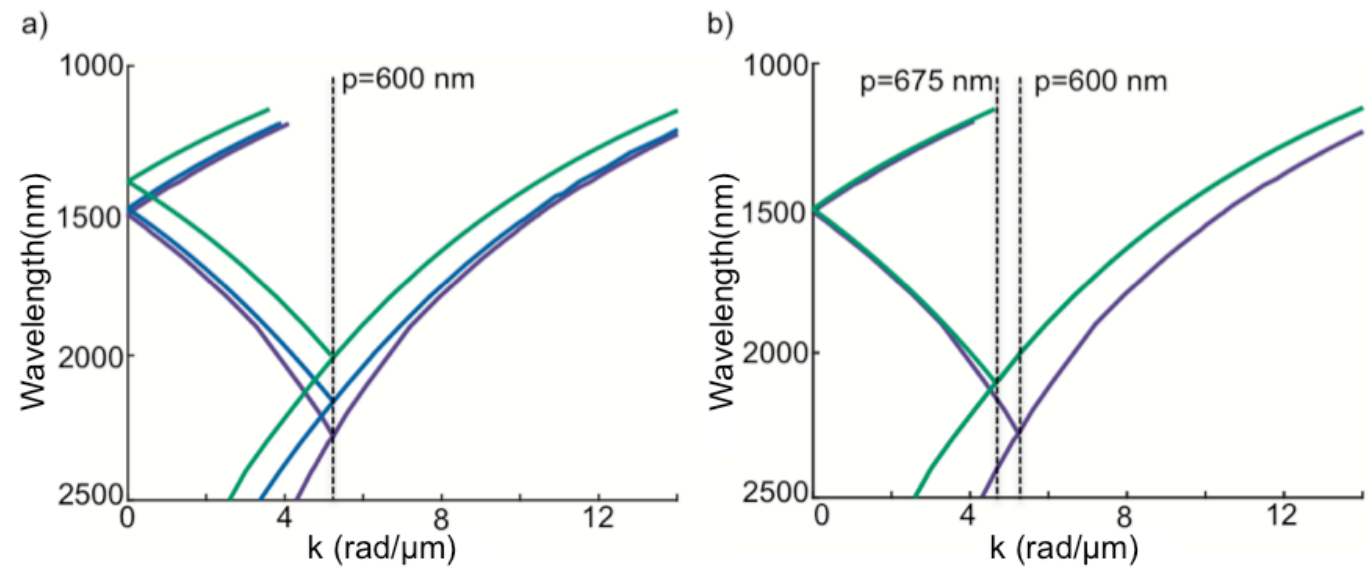

Figure S2: a) Dispersion for silicon bars in the metalens with $w=219 \mathrm{~nm}$ (purple), 205 $\mathrm{nm}$ (blue), and $158 \mathrm{~nm}$ (green), as well as band folding associated with a period of 600 
$\mathrm{nm}$, b) Dispersion plots found for silicon bars with $w=219 \mathrm{~nm}$ (purple) and $158 \mathrm{~nm}$ (green), and band folding associated with a period of $600 \mathrm{~nm}$ and $675 \mathrm{~nm}$ respectively.

In the $3 \mathrm{D}$ simulations, the periodic nature of the notched nanobars in the y-direction was enabled through Floquent boundary conditions. A port boundary condition in the zdirection below the metasurface accounted for a y-polarized, Gaussian incident field according to the equation $\mathrm{E}_{\mathrm{y}}=\mathrm{E}^{*} \exp \left(-(\mathrm{x} / 5000 \mathrm{~nm})^{2}\right)$. Scattering boundary conditions were used in the $\mathrm{x}$-direction and in the z-direction above the metasurface. Our Q factors were calculated by fitting the curves to an asymmetric Lorentzian, as follows fit $(\lambda)=$ $\left|a_{\mathrm{r}}+\mathrm{i} * \mathrm{a}_{\mathrm{i}}+\frac{-\mathrm{i} * \mathrm{c} * \gamma}{\frac{2 \pi}{\lambda}-\frac{2 \pi}{\lambda_{0}}+\mathrm{i} * \gamma}\right|^{2}$ where $\mathrm{a}_{\mathrm{r}}$ is the real background coefficient, $\mathrm{a}_{\mathrm{i}}$ is the imaginary background coefficient, $\mathrm{c}$ is a correction coefficient, $\lambda_{0}$ is the resonant wavelength, and $\gamma$ is the displacement coefficient; then the resonant wavelength was divided by the resulting linewidth of the resonance.

In the linear regime, both 2D and 3D simulations were performed by taking the refractive indices of air $(\mathrm{n}=1)$ and the $\mathrm{Si}$ as constant $(\mathrm{n}=3.4)$.

\section{Supporting Information Section 2: Guided Mode Resonance Origin in Slab Waveguide}

Before going on to investigate the high-Q behavior of our fully 3D metalens, we will further discuss the fundamental features of a guided mode resonance. Considering a 2D slab waveguide - a lower dimensional analog to the nanobars we use to construct our 
metasurface. We consider a slab $200 \mathrm{~nm}$ thick with Floquent boundaries on either side of a nanobar and port boundaries, polarized into the plane, above and below the slab. The resulting field plot for the unnotched low-Q and notched high-Q results are shown in Figure S3a and b respectively. Additionally the Transmittance plot in Figure S3c shows the background Fabry-Perot fringes found in the unperturbed case, as well as the sharp resonance that appears when the slab waveguide is notched.

We also briefly examine the specific features of the resonance as a function of the notch geometry. As previously discussed in context of the guided mode dispersion above, the perturbation period has the strongest effect on the spectral position of the resonance. In Figure S3d the notch period is varied from $500 \mathrm{~nm}$ to $650 \mathrm{~nm}$, and the resonance is shown to red-shift by approximately $105 \mathrm{~nm}$ with a $50 \mathrm{~nm}$ shift in the period. Changes in the dimensions of the notch width and depth also change the resonances spectral position, red shifting with increasing width and decreasing depth. The depth of the perturbation also most significantly affects the strength of the quality factor, as considered by Figure S3g. The shallower the perturbation, the stronger the resulting $Q$ factor, as the system is more similar to the continuous waveguide with infinite $\mathrm{Q}$. 
a)
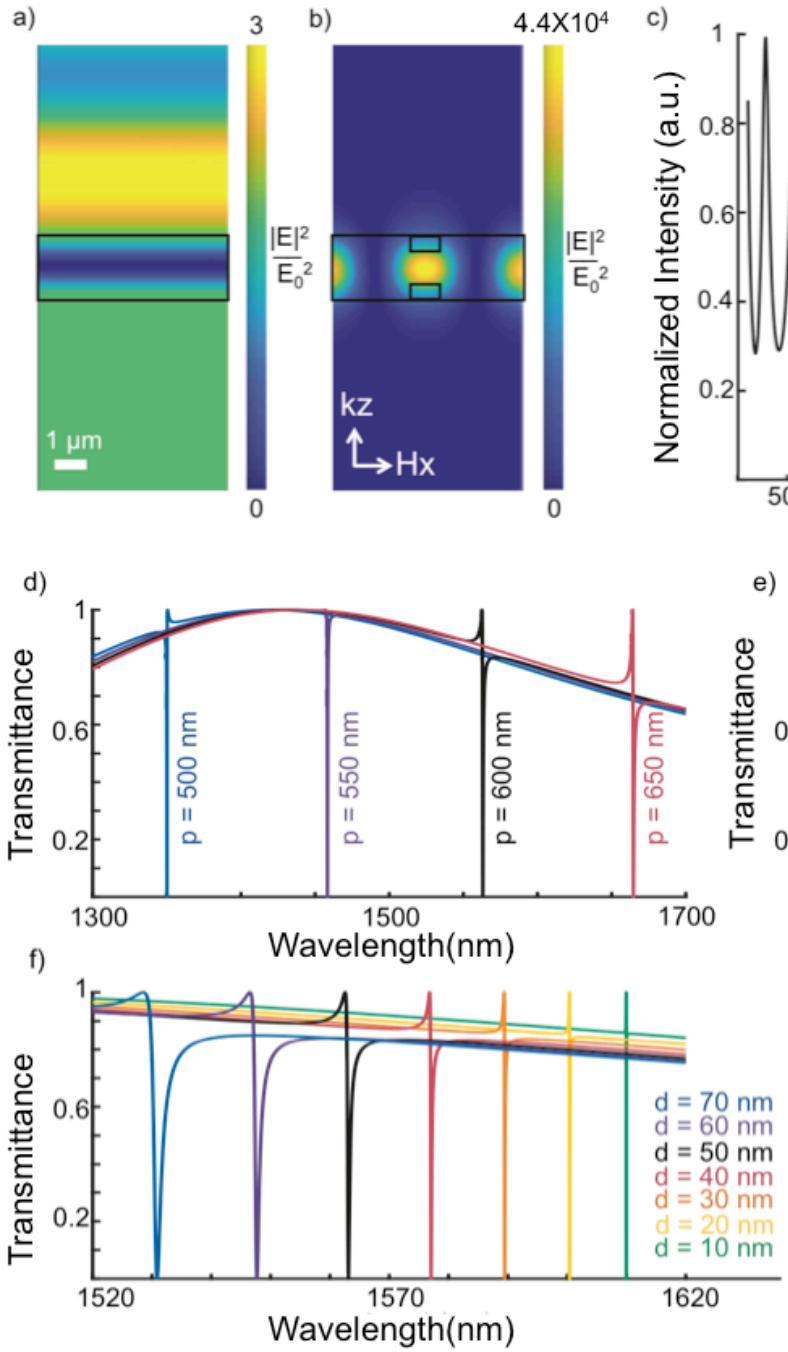

e)
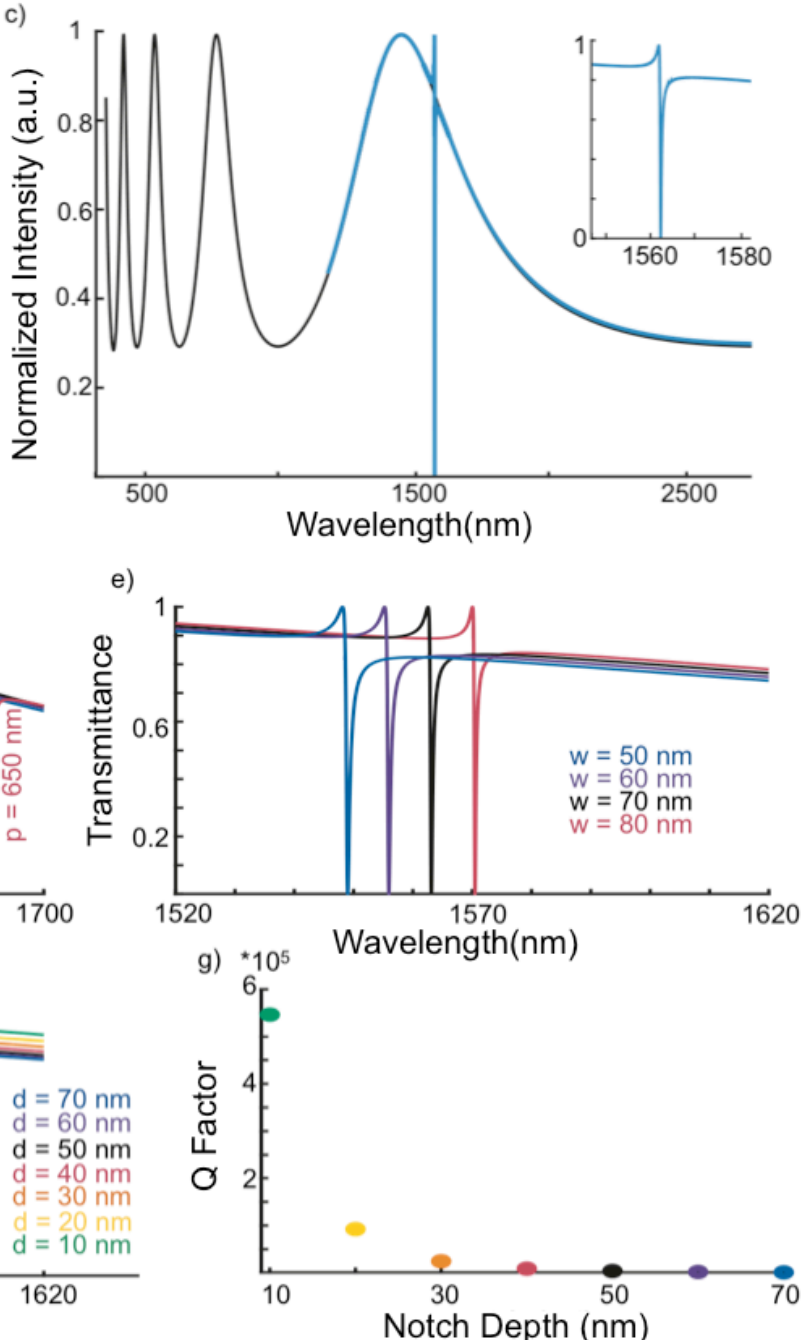

Figure S3: a) $|E(r)|^{2}$ field plot for a $200 \mathrm{~nm}$ slab waveguide excited at $\lambda=1563 \mathrm{~nm}$ and normalized to the incident plane wave. b) $|E(r)|^{2}$ field plot for a $200 \mathrm{~nm}$ notched slab waveguide with a period of $600 \mathrm{~nm}$, notch width of $70 \mathrm{~nm}$ and depth of $50 \mathrm{~nm}$, excited at $\lambda=1563 \mathrm{~nm}$ and normalized to the incident plane wave. c) Transmittance spectrum for the $200 \mathrm{~nm}$ unnotched (black) and notched (blue) slab waveguide, zoom in on the resonance for the notched case is plotted in inset. d) Transmittance spectrum for the $200 \mathrm{~nm}$ notched waveguide with the notch period varying as $500 \mathrm{~nm}$ (blue), $550 \mathrm{~nm}$ (purple), $600 \mathrm{~nm}$ 
(black), and $650 \mathrm{~nm}$ (red). e) Transmittance spectrum for the $200 \mathrm{~nm}$ notched waveguide with the notch width varying as $50 \mathrm{~nm}$ (blue), $60 \mathrm{~nm}$ (purple), $70 \mathrm{~nm}$ (black), and $80 \mathrm{~nm}$ (red). f) Transmittance spectrum for the $200 \mathrm{~nm}$ notched waveguide with the notch depth varying as $70 \mathrm{~nm}$ (blue), $60 \mathrm{~nm}$ (purple), $50 \mathrm{~nm}$ (black), $40 \mathrm{~nm}$ (red), $30 \mathrm{~nm}$ (orange), 20 $\mathrm{nm}$ (yellow), and $10 \mathrm{~nm}$ (green). g) Quality factor of the resonance as a function of notch depth.

Further, we investigate the robustness of this resonance to perturbation inconsistencies, as future experimental investigations of this system may introduce instabilities during fabrication, such as the finite length of the nanobars and etching inconsistencies. Figure S4a investigates non-ideal notches, with rounded edges, and shows that as the radius of curvature increases the resonance blue-shifts. We also look at several cases of imperfect repetition of the dimensions of the notches. In Figure S4b the pairs of notches are offset from one another, and as this offset increases the resonance red-shifts. Finally in Figure S4c, we consider several periods of notches, where the repetition of the notches is not perfectly equal to the desired perturbation period. In the most extreme case considered, this is shown to cause other spectral features to appear, but in all cases the main resonance's quality factor remains robust. 

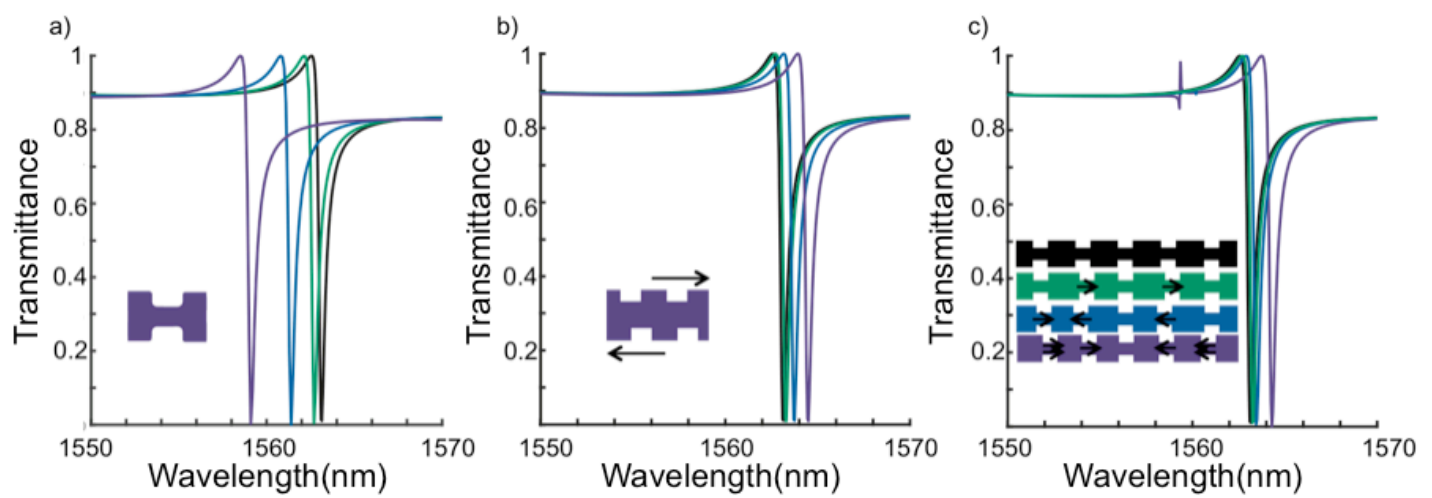

Figure S4: a) Transmittance spectrum for the $200 \mathrm{~nm}$ notched waveguide considering the notches with curved corners with the radius of curvature varying as $0 \mathrm{~nm}$ (black), $\mathrm{nm} 10$ (green), $20 \mathrm{~nm}$ (blue), and $30 \mathrm{~nm}$ (purple) as shown in the inset. b) Transmittance spectrum for the $200 \mathrm{~nm}$ notched waveguide considering the top and bottom notches offset from one another $0 \mathrm{~nm}$ (black), $10 \mathrm{~nm}$ (green), $20 \mathrm{~nm}$ (blue), and $30 \mathrm{~nm}$ (purple) as shown in the inset. c) Transmittance spectrum for the $200 \mathrm{~nm}$ notched waveguide considering the notch period varying inconsistently as shown by the associated colors in the inset, single shifts are equal to $10 \mathrm{~nm}$ while double shifts are $20 \mathrm{~nm}$.

\section{Supporting Information Section 3: Nonlinear Metasurface Details}

To analyze the nonlinear behavior of our lens, 3D simulations integrated the equation for the optical Kerr effect $\varepsilon(r)=\varepsilon_{0}\left(\varepsilon_{r}+\chi^{(3)}|E(r)|^{2}\right)$, with $\chi^{(3)}$ of $2.8^{*} 10^{-18} \mathrm{~m}^{2} / \mathrm{V}^{2}$, into the wave equation for the Si of the metasurface. A series of simulations of the metalens, with notches in the second from the center nanobars with a period of $600 \mathrm{~nm}$, were performed at a wavelength of $1499.4 \mathrm{~nm}$ for incident intensities varying from 0.01 
$\mathrm{mW} / \mathrm{um}^{2}$ to $1 \mathrm{~mW} / \mathrm{um}^{2}$. Figure $\mathrm{S} 5 \mathrm{~b}$ illustrates the intensity at the focal spot, $4 \mu \mathrm{m}$ from the metalens, for all input intensities, including both the normalized and unnormalized (inset) values.

Figure S5b shows the change of the maximum focal spot position with input intensity. Similar to the results shown in the main text, these results illustrate the power-limiting behavior of the lens and the shift in the focal length at higher input powers. FigS5c shows the focal behavior of the metalens at various powers normalized to their own maximum; this plot clearly shows not only the change in the maximum of the focal spot, but also the gradual shift in the overall focal spot area with increasing focal intensity.
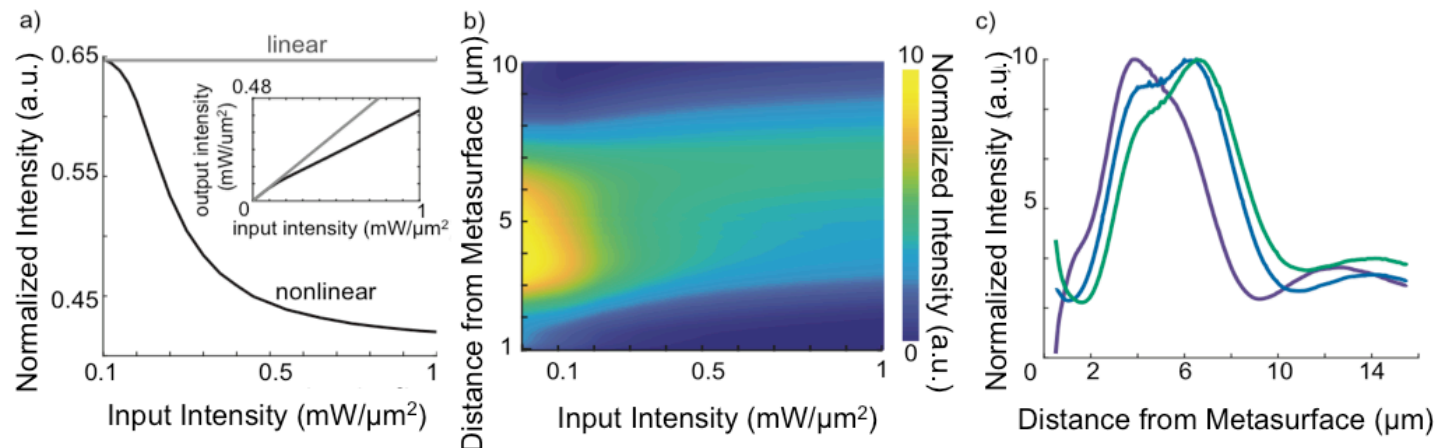

Figure S5: a) Normalized intensity values at a plane $4 \mu \mathrm{m}$ from the metasurface for the linear (grey) and nonlinear (black) metalens, inset shows unnormalized case b) Color map of intensity in the focal spot plotted as the distance from metasurface for input intensities from $0.01 \mathrm{~mW} / \mu \mathrm{m}^{2}$ to $1 \mathrm{~mW} / \mu \mathrm{m}^{2}$, c) Focal response in the propagation direction (along the z-axis above the high-Q metasurface) for various input intensities normalized to their own maximum intensity value, showing a focal length shift of the focal spot. 


\section{$\underline{\text { Supporting Information Section 4: Multi-Resonant Lens Simulations }}$}

Similar to the single notched metasurface lens design, the metalens with three distinct sets of notched nanobars depicts a redshift of its high-Q resonance as power is increased from $0.1 \mathrm{~mW} / \mu \mathrm{m}^{2}$ (purple) to $0.5 \mathrm{~mW} / \mu \mathrm{m}^{2}$ (blue) shown in Figure S6. For the notched nanobar second from the center, the peak of the high-Q resonance shifts from $1499.4 \mathrm{~nm}$ to $1499.6 \mathrm{~nm}$. For the notched nanobar third from the center, the peak of the high-Q resonance shifts from $1480.9 \mathrm{~nm}$ to $1481.1 \mathrm{~nm}$. For the notched nanobar fourth from the center, the peak of the high-Q resonance shifts from $1388.45 \mathrm{~nm}$ to $1388.65 \mathrm{~nm}$.

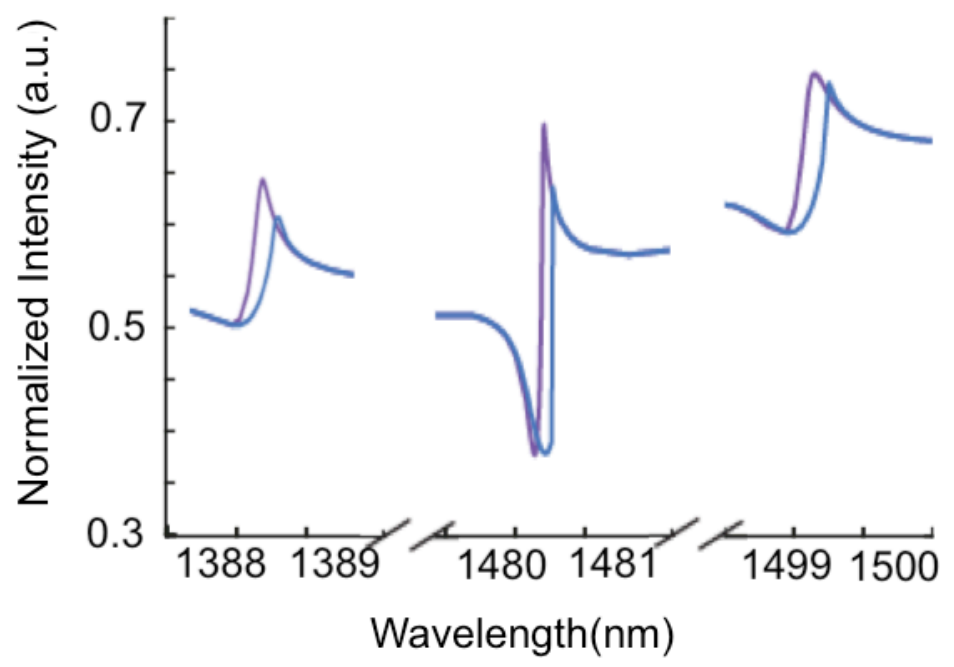

Figure S6: Spectral features calculated at the xy plane 4 m from the metasurface for input intensity of $0.1 \mathrm{~mW} / \mu \mathrm{m}^{2}$ (purple) and $0.5 \mathrm{~mW} / \mu \mathrm{m}^{2}$ (blue) for the multi-notched metalens 
Finally, we show here the low-power $\left(0.1 \mathrm{~mW} / \mu \mathrm{m}^{2}\right)$ and higher-power $\left(0.5 \mathrm{~mW} / \mu \mathrm{m}^{2}\right)$ field intensity plots for the resonance that appears at $1388.45 \mathrm{~nm}$. As with the other resonances in the multi-resonant metalens, power-limiting-like behavior can be seen at the intended focal spot near $4 \mathrm{um}$. However, as this resonance occurs far from the intended operating wavelength of $1500 \mathrm{~nm}$, the overall lensing behavior of the metalens at this wavelength is poor. This nonetheless shows the robustness of the high-Q nature, decoupled from the lensing response.
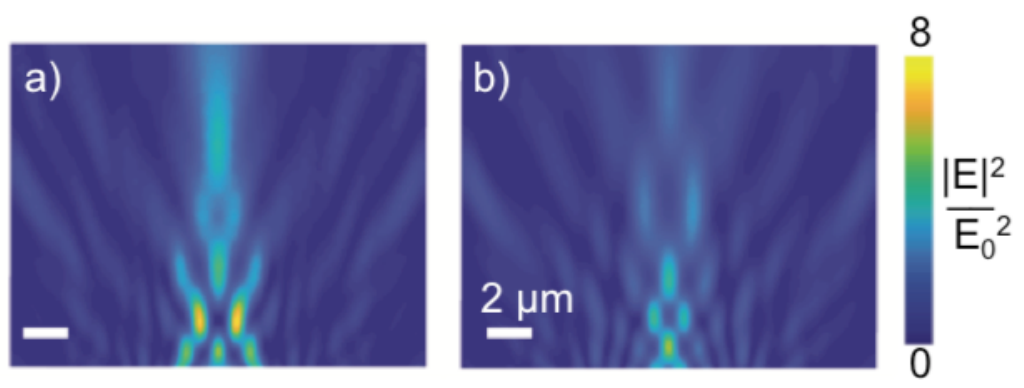

Figure S7: $|E(r)|^{2}$ field plot for the nonlinear lens excited at $\lambda=1388.45 \mathrm{~nm}$ and normalized to the mean of the input gaussian field for a) an input intensity of $0.1 \mathrm{~mW} / \mu \mathrm{m}^{2}$ and b) an input intensity of $0.5 \mathrm{~mW} / \mu \mathrm{m}^{2}$

\section{Supporting Information Section 5: Alternate High-Efficiency, High-Q Metalens}

\section{Design}

Here we briefly investigate an alternative high-Q metalens design. This metalens, utilizing the same width bars as the previous design, is modeled with incident x-polarized light (rather than y-polarized as was previously the case). An initial 2D simulation 
determined the phase delay and Transmittance, shown in Figure S8a. In this case, the Transmittance was found to be higher overall across the full 2 pi phase space. As a consequence the efficiency of the lensing overall was found to be higher, reaching a normalized intensity of 0.92 , as shown in Figure $\mathrm{S} 8 \mathrm{~b}$. This result further shows how the specifics of the metalens design can be optimized independently from the high-Q nature and modulation technique.
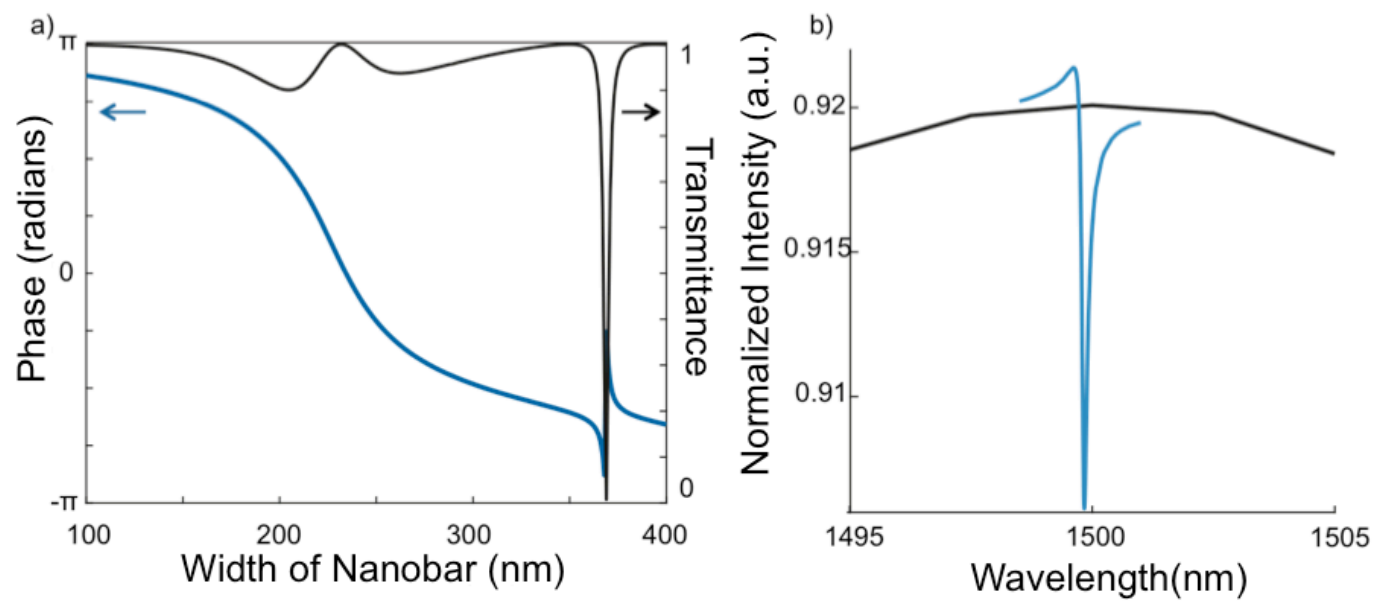

Figure S8: a) Transmittance (black) and phase shift (blue) associated with a normally incident, $x$-polarized plane wave on a series of bars of height $700 \mathrm{~nm}$, spaced $700 \mathrm{~nm}$ apart, with width varying from 100 to $400 \mathrm{~nm}$ b) Spectra for an unnotched (black curve) and notched (blue curve) metalens excited by $x$-polarized light calculated for the xy plane

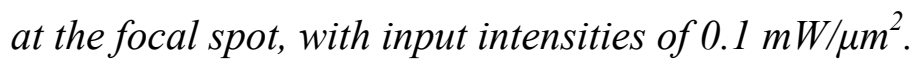

\section{Supporting Information Section 6: Influence of Another Nonlinear Effect}


In the main text we only considered the nonlinear Kerr effect's influence on the permittivity of our nanobars. However, there are other nonlinear processes that could occur and influence the permittivity of our silicon as a function of optical intensity. In the following we briefly explore the effects of Two Photon Absorption (TPA), a nonlinear process that leads to an increase in absorption as the optical intensity increases. In our full field simulations we account for TPA by introducing an imaginary part to the nonlinear susceptibility. The absolute value of the nonlinear susceptibility sets the power threshold for the onset of nonlinear behavior, whereas the figure of merit, $(\mathrm{FOM})=\operatorname{Re}(\chi(3)) / 4 \pi$ $\operatorname{Im}(\chi(3))$, accounts for potential level of absorption due to TPA In Figure S9, the results of S2a have been recalculated while incorporating a non-zero TPA effect. As clearly shown for representative FOM values varying from 0.5 to 5 , the deleterious nonlinear effect does not influence the nonlinear red-shift of the high-Q resonance. While it does affect the peak of the resonance, and thus will somewhat influence the specific high-Q strength, the Q strength is nonetheless still significantly higher than would be seen for a standard metalens. As such, we do not believe that TPA will be a significant barrier to realizing the present high-Q metalens scheme. 


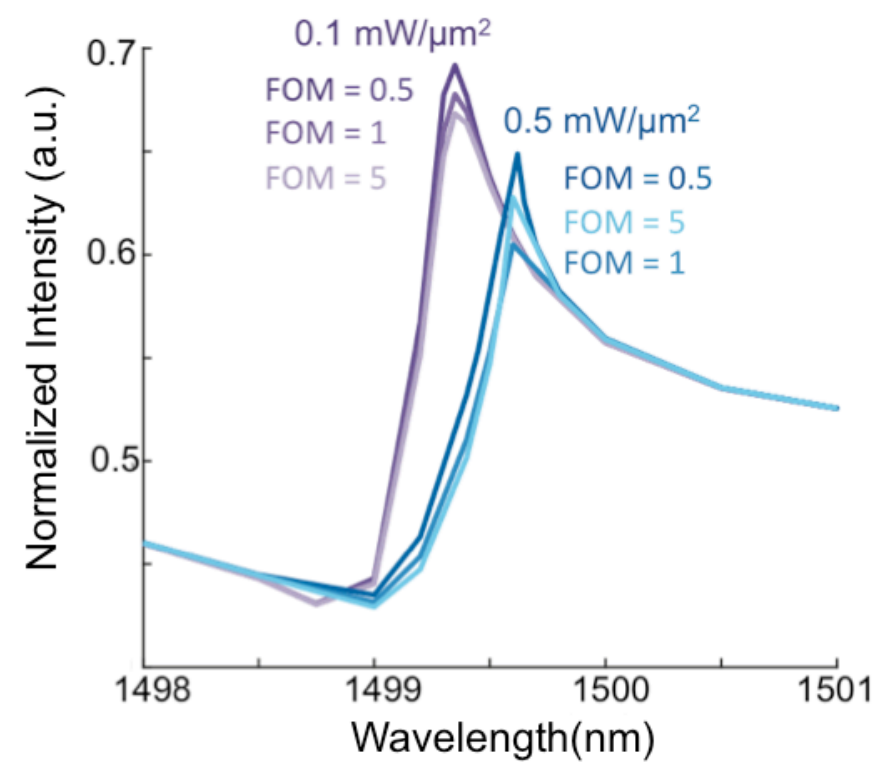

Figure S9: Spectra calculated at the focal spot for input intensities of $0.1 \mathrm{~mW} / \mu \mathrm{m}^{2}$ (purple) and $0.5 \mathrm{~mW} / \mu \mathrm{m}^{2}$ (blue), calculated with varying levels of Two Photon Absorption accounted for. 